\title{
Laminar flow and heat transfer characteristics of interrupted microchannel heat sink with ribs in the transverse
}

\section{microchambers}

\author{
Lei Chai ${ }^{\mathrm{a},}$, Guo Dong Xia ${ }^{\mathrm{b}}$, Hua Sheng Wang ${ }^{\mathrm{a}}$ \\ ${ }^{\text {a }}$ School of Engineering and Materials Science, Queen Mary University of London, Mile End Road, London E1 4NS, UK \\ ${ }^{\mathrm{b}}$ Key Laboratory of Enhanced Heat Transfer and Energy Conservation, Ministry of Education, College of Environmental and \\ Energy Engineering, Beijing University of Technology, Beijing, China
}

\begin{abstract}
The paper is focused on the investigation of the laminar flow and heat transfer characteristics in the interrupted microchannel heat sink with ribs in the transverse microchambers. A three-dimensional model based on finite volume approach and SIMPLEC algorithm is performed considering entrance effect, conjugate heat transfer, viscous heating and temperature-dependent thermo-physical properties. Five different rib configurations are considered, including rectangular, backward triangular, diamond, forward triangular and ellipsoidal. The present study firstly examines the effect of such ribs on velocity contour, pressure distribution and temperature distribution, further the local pressure drop and heat transfer characteristics in such microchannel heat sinks. Result shows that the ribs in the transverse microchambers can effectively prevent the decline of local heat transfer coefficient along the flow direction. Secondly, the average friction factor and Nusselt number for such microchannel heat sinks have been studied. Results show that for Reynolds number ranging

\footnotetext{
${ }^{*}$ Corresponding author. Tel.: +44 2078827306.

E-mail address: 1.chai@qmul.ac.uk (Lei Chai).
} 
from 187 to 715 , there is a $24-57 \%$ increase in the Nusselt number for the interrupted microchannel heat sinks with ribs, while the friction factor increased by $3-70 \%$, comparing with the straight microchannel heat sink. Finally, based on the performance evaluation criteria, the performance of such microchannel heat sink has been comprehensively evaluated. Results shows that for the studied Reynolds number range and microchannel geometries in this paper, the interrupted microchannel heat sink with ellipsoidal ribs in the transverse microchambers shows the best heat transfer performance, with maximum 1.39 of performance evaluation criteria.

Key words interrupted microchannel; numerical simulation; pressure drop; heat transfer; performance evaluation criteria

\section{Introduction}

Microchannel heat sinks are currently widely used for the high heat flux applications such as electronics cooling, due to their advantages such as compactness, light weight and higher heat transfer surface area to fluid volume ratio compared with other macroscale systems [1]. Moreover, several techniques of enhancing the heat transfer performance of micro/minichannel heat exchanger have been studied, in order to deal with the cooling problems of electric power and electronic equipment with high heat flux [2]. Among these methods, to interrupt the thermal boundary layer, to induce the mainstream separation, and to enhance mixing of cold and hot fluid are significant means to enhance heat transfer in microscale [3]. 
In recent years, $\mathrm{Xu}$ et al. $[4,5]$ have experimentally and numerically investigated the interrupted microchannel heat transfer enhancement using the thermal boundary layer redeveloping concept. Their interrupted microchannel heat sink consisted of a set of separated zones adjoining shortened parallel microchannels and transverse microchambers (the regions between the separated parallel microchannel zones). The transverse microchamber was used to separate the whole flow length into several independent zones. They found that the computed hydraulic and thermal boundary layers were redeveloping in each separated zone due to the shortened flow length for the interrupted microchannel heat sink, and the periodic thermal developing flow was responsible for the significant heat transfer enhancement. Chai et al. $[6,7]$ experimentally and numerically studied the pressure drop and heat transfer characteristics of microchannel heat sinks with periodic expansion-constriction cross-sections. Each heat sink consisted of 10 parallel microchannels with $0.1 \mathrm{~mm}$ wide and $0.2 \mathrm{~mm}$ deep in constant cross-section region and each microchannel consisted of an array of periodic expansion-constriction cross-sections (fan-shape and triangular reentrant cavities). Their results indicated that their proposed microchannel heat sinks could significantly improve heat transfer performance with an acceptable pressure drop compared to the bare straight microchannel heat sink and the heat transfer enhancement mechanisms could attribute to the interruption of boundary layer formation, establishment of secondary flow and provision of more surface area. Xia et al. $[8,9]$ numerically investigated the effect of geometric parameters on water flow and heat transfer characteristics in two types of microchannel heat sink, respectively with fan-shape and triangular reentrant cavities. The studied geometric parameters included the length, width and height of the reentrant cavity, and the space 
between two adjoining reentrant cavities. They found that these geometric parameters had a significant influence on the pressure drop and heat transfer characteristics. Based on the performance evaluation criteria, the optimal geometric parameters were obtained and the maximum 1.62 could be reached comparing with the straight microchannel heat sink. Cheng [10] numerically studied the laminar flow and heat transfer in a stacked two-layer microchannel heat sink with multiple passive microstructures. He found that the enhanced mixing mechanism of cold and hot fluid could lead to better heat transfer and lower thermal resistance. Therefore, the stacked microchannel with passive structures had better heat transfer performance than the smooth microchannel. Abed et al. [11] carried out a combined experimental and numerical investigation to study the characteristics of laminar flow and forced convection heat transfer in a square cross-section wavy serpentine microchannel with the upper wall insulated and other side walls held at constant temperature. They found that the growth of secondary-flow vortices promoted fluid mixing in the serpentine microchannel and led to an enhancement of the convective heat transfer relative to a straight microchannel. Hong and Cheng [12] numerically investigated the laminar forced convection of water in offset strip-fin microchannel network heat sinks for microelectronic cooling, with a three-dimensional mathematical model consisting of N-S equations and energy conservation equation with the conjugate heat transfer between the heat sink base and liquid coolant. They found that due to the periodical change of the flow direction, the convective heat transfer was enhanced by mixing the cold and hot coolant, and the periodical breakup of boundary layer was another factor to enhance heat transfer. Foong et al. [13] presented a three-dimensional numerical simulation to investigate the fluid flow and heat transfer characteristics of a square 
microchannel with four longitudinal internal fins. They concluded that the internal fins in a microchannel had the potential to provide heat transfer augmentation. The internal fins could lead to the development of the thermal boundary layer, better flow mixing, steeper velocity gradient at the heated surface, and increased surface heat transfer coefficient and Nusselt number. Ebrahimi et al. [14] numerically investigated the liquid flow and conjugated heat transfer performance of single phase laminar flow in rectangular microchannels equipped with longitudinal vortex generators (LVGs). Five different configurations of the microchannel with different angles of attack of the LVGs were considered. The results showed that for Reynolds number ranged from 100 to 1100 , there was a $2-25 \%$ increase in the Nusselt number for microchannels with LVGs, while the friction factor increased by $4-30 \%$.

Based on the methods used for heat transfer enhancement in microchannels mentioned in the above literatures, Chai et al. [3] introduced the staggered rectangular ribs into the transverse microchambers for the interrupted microchannel, considering the rectangular rib not only induced the mainstream separation, but also enhanced the convective fluid mixing of cold and hot fluid. Further, Chai et al. [15] introduces the offset ribs to the two opposite channel sidewalls for rectangular microchannel heat sink, with the purposes to provide shorter length required for repeated thermal developing flow, to work as micromixers or baffles for better mixing of cold and hot fluid, to be easily manufactured by micro machining method, and with higher reliability than the active measures. They found that different offset ribs resulted in different heat transfer performance. The microchannel heat sink with forward triangular offset ribs performed best as Reynolds numbers lower than 350, and the one with semicircular offset ribs yielded the best performance as Reynolds numbers higher than 400. In 
order to obtain the insight into the fluid flow and heat transfer mechanisms and present a systematic and detailed analysis for the interrupted microchannel heat sink, the present paper focuses on the different rib configurations in the transverse microchambers for the interrupted microchannel heat sink, including rectangular, backward triangular, diamond, forward triangular and ellipsoidal, to check their influences on pressure drop and heat transfer characteristics.

\section{Computational method}

\subsection{Conservation equations}

In order to simplify the modelling simulation, the following assumptions are employed: (1) developing flow and heat transfer in microchannels, (2) steady, laminar flow and heat transfer, (3) varied fluid thermal-physical properties, (4) axial thermal conduction, (5) viscous dissipation. For the conjugate heat transfer problem, combining heat conduction in the solid and convection to the cooling fluid, the governing equations in the Cartesian tensor form are:

Continuity equation

$\frac{\partial}{\partial x_{i}}\left(\rho u_{i}\right)=0$

Momentum equation

$$
\frac{\partial}{\partial x_{i}}\left(\rho_{\mathrm{f}} u_{i} u_{j}\right)=-\frac{\partial p}{\partial x_{j}}+\frac{\partial}{\partial x_{i}}\left[\mu_{\mathrm{f}}\left(\frac{\partial u_{j}}{\partial x_{i}}+\frac{\partial u_{i}}{\partial x_{j}}\right)\right]
$$

Energy equation

$$
\frac{\partial}{\partial x_{i}}\left(\rho_{\mathrm{f}} u_{i} c_{\mathrm{pf}} T\right)=\frac{\partial}{\partial x_{i}}\left(k_{\mathrm{f}} \frac{\partial T}{\partial x_{i}}\right)+\mu_{\mathrm{f}}\left[2\left(\frac{\partial u_{i}}{\partial x_{i}}\right)^{2}+\left(\frac{\partial u_{j}}{\partial x_{i}}+\frac{\partial u_{i}}{\partial x_{j}}\right)^{2}\right]
$$


For the solid $\quad \frac{\partial}{\partial x_{i}}\left(k_{\mathrm{s}} \frac{\partial T}{\partial x_{i}}\right)=0$

where $\rho$ is density, $\mu$ is dynamic viscosity, $c_{\mathrm{p}}$ is specific heat capacity, $k$ is thermal conductivity, $x_{1}, x_{2}$ and $x_{3}$ are $x, y$ and $z$ coordinates, respectively, as shown in Fig. 1a. Subscripts $f$ and s refer to fluid and solid, respectively.

\subsection{Computational domain and boundary conditions}

For the periodic structure of the microchannel heat sinks along the transverse direction $(y$ direction in Fig. 1), Chai et al. [16] developed two three-dimensional heat transfer models to simulate laminar flow and heat transfer, one with single channel and the other with the whole heat sink as the computational domain. They found that there was just a little difference for pressure drop and heat transfer between the two models. Further, for reducing the number of the grid points and the calculation time for the periodic structure, Cheng [10], Sakanova et al. [17] and Xie et al. [18] used the single channel as the computational domain. From literature [3], it can be seen that the interrupted microchannel heat sinks have the periodic structure along the transverse direction, so to save the computation time and take advantage of symmetry boundary condition, a control volume containing a single microchannel along the flow direction ( $x$ direction in Fig. 1) and surrounding solid along with the base is selected for developing the present fluid flow and heat transfer model. Fig. 1a illustrates the computational domain, corresponding coordinate system and key notations used. The length, width and height of the computational domain are $10 \mathrm{~mm}, 0.25 \mathrm{~mm}$ and $0.35 \mathrm{~mm}$, respectively. The computational domain is divided into front, middle and rear microchannels with two microchambers located in between. The width and height in the microchannel region 
are $0.1 \mathrm{~mm}$ and $0.2 \mathrm{~mm}$, forming the hydraulic diameter of $133.33 \mu \mathrm{m}$. The front, middle and rear microchannels have the same length of $2.6 \mathrm{~mm}$. The transverse microchamber has the length of $1.1 \mathrm{~mm}$ and the same depth as that of the longitudinal microchannels. The staggered ribs are located in the center of transverse microchambers. Fig. 1b shows the detailed geometry parameters of the staggered ribs. Five different rib configurations are considered: rectangular, backward triangular, diamond, forward triangular and ellipsoidal. The five new interrupted microchannel heat sinks are respectively named for short as IMCHS-R, IMCHS-BT, IMCHS-D, IMCHS-FT and IMCHS-E. All the staggered ribs are $0.5 \mathrm{~mm}$ in $x$-direction, $0.1 \mathrm{~mm}$ in $y$-direction and $0.2 \mathrm{~mm}$ in $z$-direction. To determine the changes of flow structure, pressure drop and heat transfer, a straight microchannel heat sink without transverse microchamber (MCHS for short) is selected as baseline and an interrupted microchannel heat sink without ribs in the transverse microchambers (IMCHS for short) is used for comparison.

The associated boundary conditions are set as follows. The fluid velocity at the microchannel inlet is assumed uniform

$x=0: u_{\mathrm{f}}=u_{\text {in }}$ and $T_{\mathrm{f}}=T_{\text {in }}$

where $u_{\text {in }}$ and $T_{\text {in }}$ are the given fluid velocity and temperature at the channel inlet. At the channel outlet, a pressure-outlet boundary condition is applied

$x=10 \mathrm{~mm}: p_{\mathrm{f}}=p_{\text {out }}$

where $p_{\text {out }}$ is the given pressure. For the inner wall/ fluid contact surface,

$u=v=w=0,-k_{\mathrm{s}} \frac{\partial T_{\mathrm{s}}}{\partial n}=-k_{\mathrm{f}} \frac{\partial T_{\mathrm{f}}}{\partial n}$

where $n$ is the local coordinate normal to the wall. At the substrate of computational domain, 
$z=0:-k_{\mathrm{s}} \frac{\partial T_{\mathrm{s}}}{\partial z}=q$

At the two sides of the computational domain,

$y=0, \frac{\partial T_{s}}{\partial y}=0$

$y=0.25 \mathrm{~mm}, \frac{\partial T_{\mathrm{s}}}{\partial y}=0$

For other surfaces,

$-k_{\mathrm{f}} \frac{\partial T_{\mathrm{f}}}{\partial x}=0$ or $-k_{\mathrm{s}} \frac{\partial T_{\mathrm{s}}}{\partial x}=0$

The parameters used in this study are in the following ranges: $u_{\text {in }}=1-5 \mathrm{~m} \cdot \mathrm{s}^{-1}, T_{\text {in }}=293 \mathrm{~K}$, $p_{\text {out }}=0$ (gauge pressure) and $q=10^{6} \mathrm{~W} \cdot \mathrm{m}^{-2}$. The fluid and solid are deionized water and silicon, respectively. The thermo-physical properties of water including $\rho_{\mathrm{f}}, \mu_{\mathrm{f}}, c_{\mathrm{pf}}$ and $k_{\mathrm{f}}$ depend on temperatures in accordance to Incropera [19], and the thermal conductivity of silicon $k_{\mathrm{s}}$ is set as a constant of $148 \mathrm{~W} \cdot \mathrm{m}^{-1} \cdot \mathrm{K}^{-1}$ in the computations.

\subsection{Solution methods and convergence criteria}

The governing equations were solved using the finite volume based computational fluid dynamics solver ANSYS FLUENT 12.0. The SIMPLEC algorithm is applied to solve the governing differential equations for the velocity, pressure and temperature fields in the control volume. The standard discretization scheme is used in the modeling following the second order upwind of the momentum and energy discretization. For the interrupted microchannels, an unstructured mesh based on rectangular grid elements is utilized. The solutions were considered to be converged when the normalized residual values were less than $10^{-6}$ for all variables. The numerical code is verified in a number of ways to ensure the validity. For every microchannel heat sink, a grid independence test is conducted using several different mesh 
sizes. For example, for the IMCHS-R with rib length $0.4 \mathrm{~mm}$, as shown in Chai et al. [3], as $u_{\text {in }}=4 \mathrm{~m} \cdot \mathrm{s}^{-1}$, the deviations of average friction factor using $0.267,0.417$ and 0.652 million grids from that of 1.019 million grids are $9.31 \%, 4.19 \%, 1.97 \%$ and $0.43 \%$ respectively, and the deviations of Nusselt number using $0.267,0.417$ and 0.652 million grids from that of 1.019 million grids are $10.12 \%, 6.24 \%, 2.49 \%$ and $0.76 \%$, respectively. Thus the 0.652 million grids is used for this mentioned interrupted microchannel heat sink. Using the same method, the grid points are determined for every studied microchannel heat sink. The 0.692 , 0.691, 0.692, 0.691 and 0.693 million grids are respectively used for IMCHS-R, IMCHS-BT, IMCHS-D, IMCHS-FT and IMCHS-E and Fig. 2 shows the grid in the plane of $z=0.25 \mathrm{~mm}$.

\subsection{Data acquisition}

Parameters characterizing the fluid flow and heat transfer in the microchannels are of interest in the present work. The Reynolds number is defined as

$$
R e=\frac{\rho_{\mathrm{f}} u_{\mathrm{m}} D_{\mathrm{h}}}{\mu_{\mathrm{f}}}
$$

where $\rho_{\mathrm{f}}$ is the volume average fluid density, $\mu_{\mathrm{f}}$ is the mass average fluid dynamic viscosity. Ignoring the difference in the microchambers due to the changes in the flow passages, the average flow velocity $u_{\mathrm{m}}$ and the hydraulic diameter $D_{\mathrm{h}}$ are calculated based on the straight microchannel for the whole length, using the same definition as $\mathrm{Xu}$ et al. [4, 5] for the interrupted microchannels with the purpose of easy to compare and study the effect of microchamber in laminar flow and heat transfer characteristics. The $\rho_{\mathrm{f}}$ and $\mu_{\mathrm{f}}$ are defined as

$$
\rho_{\mathrm{f}}=\frac{\int \rho_{\mathrm{f}, \mathrm{i}} d V}{\int d V}
$$


$\mu_{\mathrm{f}}=\frac{\int \mu_{\mathrm{f}, \mathrm{i}} \rho_{\mathrm{f}, \mathrm{d}} d V}{\int \rho_{\mathrm{f}, \mathrm{i}} d V}$

The local Fanning friction factor is given by

$f_{\mathrm{x}}=\frac{\left(p_{\mathrm{in}}-p_{\mathrm{x}}\right) D_{\mathrm{h}}}{2 \rho_{\mathrm{f}} x u_{\mathrm{m}}{ }^{2}}$

where $p_{\text {in }}$ is the mass-weighted average pressure in the microchannel inlet and $p_{\mathrm{x}}$ is the pressure in the $x$ location. The $p_{\text {in }}$ and $p_{\mathrm{x}}$ are defined as

$$
\begin{aligned}
& p_{\text {in }}=\frac{\int p_{\mathrm{in}, \mathrm{i}} \rho_{\mathrm{f}, \mathrm{i}} u_{\mathrm{f}, \mathrm{i}} d A}{\int \rho_{\mathrm{f}, \mathrm{i}} u_{\mathrm{f}, \mathrm{i}} d A} \\
& p_{\mathrm{x}}=\frac{\int p_{\mathrm{x}, \mathrm{i}} \rho_{\mathrm{f}, \mathrm{i}} u_{\mathrm{f}, \mathrm{i}} d A}{\int \rho_{\mathrm{f}, \mathrm{i}} u_{\mathrm{f}, \mathrm{i}} d A}
\end{aligned}
$$

The average Fanning friction factor is given by

$$
f_{\text {ave }}=\frac{\left(p_{\text {in }}-p_{\text {out }}\right) D_{\mathrm{h}}}{2 \rho_{\mathrm{f}} L u_{\mathrm{m}}{ }^{2}}
$$

where $L$ is the length of the interrupted microchannel heat sink, $p_{\text {out }}$ is the pressure in the microchannel outlet and assumed to be 0 .

The local convective heat transfer coefficient and the local Nusselt number are defined as

$$
\begin{aligned}
& h_{\mathrm{x}}=\frac{q L W}{A\left(T_{\mathrm{w}, \mathrm{x}}-T_{\mathrm{f}, \mathrm{x}}\right)} \\
& N u_{\mathrm{x}}=\frac{h_{\mathrm{x}} D_{\mathrm{h}}}{k_{\mathrm{f}}}
\end{aligned}
$$

where $q$ represents heat flux at the heat sink base, $A$ is the contact surface area of water and silicon for a single microchannel, $W$ is the width of computational domain, $k_{\mathrm{f}}$ is the mass average fluid thermal conductivity and defined as $k_{\mathrm{f}}=\frac{\int k_{\mathrm{fi}, \mathrm{f}} \rho_{\mathrm{f}, \mathrm{t}} d V}{\int \rho_{\mathrm{f}, \mathrm{i}} d V}$

$T_{\mathrm{w}, \mathrm{x}}$ and $T_{\mathrm{f}, \mathrm{x}}$ are the local heat sink base temperature and the local bulk fluid temperature, respectively, defined as 


$$
\begin{aligned}
& T_{\mathrm{w}, \mathrm{x}}=\frac{\int T_{\mathrm{w}, \mathrm{y}, \mathrm{y}} d y}{\int d y} \\
& T_{\mathrm{f}, \mathrm{x}}=\frac{\int T_{\mathrm{f}, \mathrm{x}} \rho_{\mathrm{f}, \mathrm{x}, \mathrm{x}} u_{\mathrm{f}, \mathrm{x}} d A}{\int \rho_{\mathrm{f}, \mathrm{x}, \mathrm{x}} u_{\mathrm{f}, \mathrm{x}, \mathrm{x}} d A}
\end{aligned}
$$

The average heat transfer coefficient and Nusselt number can be obtained by

$$
\begin{aligned}
& h_{\text {ave }}=\frac{1}{L} \int h_{\mathrm{x}} d x \\
& N u_{\text {ave }}=\frac{1}{L} \int N u_{\mathrm{x}} d x
\end{aligned}
$$

In order to access the enhanced heat transfer surfaces in heat exchanger design, Webb [20] outlined detailed procedures to calculate the performance improvement and to select the optimum surface geometry. For the newly proposed interrupted microchannel heat sink, the performance evaluation criteria is defined as the ratio of the heat transfer coefficient of the new interrupted microchannels $\left(h_{\mathrm{ave}}\right)$ to that of the conventional straight microchannel $\left(h_{\mathrm{ave}, 0}\right)$ at an equal pumping power. For a constant pumping power,

$(V \Delta p)_{0}=(\dot{V} \Delta p)$

The relationship between the average friction factor and Reynolds number [21] can be expressed as

$$
\begin{aligned}
& f_{\text {ave }, 0} R e_{0}^{3}=f_{\text {ave }} R e^{3} \\
& R e_{0}=\operatorname{Re}\left(f_{\text {ave }} / f_{\text {ave }, 0}\right)^{1 / 3}
\end{aligned}
$$

Therefore, the $P E C$ is given by

$$
P E C=\left.\frac{h_{\text {ave }}}{h_{\text {ave }, 0}}\right|_{\mathrm{pp}}=\left.\frac{N u_{\mathrm{ave}}}{N u_{\mathrm{ave}, 0}}\right|_{\mathrm{pp}}=\frac{N u_{\mathrm{ave}} / N u_{\mathrm{ave}, 0}}{\left(f_{\mathrm{ave}} / f_{\mathrm{ave}, 0}\right)^{1 / 3}}
$$

where $N u_{\mathrm{ave}, 0}$ and $f_{\mathrm{ave}, 0}$ respectively stand for Nusselt number and friction factor of the conventional straight microchannel. 


\section{Results and discussion}

\subsection{Model verification}

In order to verify the ability of the solver to predict accurate and reliable results, validation of the numerical code was performed against the experimental results presented by Chai et al. [3, 7] for IMCHS-R with rib length $0.4 \mathrm{~mm}$ and MCHS. Figs. $3 \mathrm{a}$ and $\mathrm{b}$ indicate the predicted $f_{\text {ave }}$ and $N u_{\text {ave }}$ comparing with the experimental results. Considering the experimental uncertainties $\left(3.95 \%\right.$ for $f_{\text {ave }}$ and $7.39 \%$ for $N u_{\text {ave }}$ of IMCHS-R with rib length $0.4 \mathrm{~mm})$, the different experiment conditions $\left(q=1.22 \times 10^{6} \mathrm{~W} \cdot \mathrm{m}^{-2}\right.$ for experiment of IMCHS-R with rib length $0.4 \mathrm{~mm}$ and $q=10^{6} \mathrm{~W} \cdot \mathrm{m}^{-2}$ for simulation) and the influence of entrance and exit plenums in the two ends of microchannel region for the experimental data (which is studied by Chai et al. [16]), good agreements are found to achieve between numerical results and the experimental results of Chai et al. [3, 7]. The maximum deviation of numerical $f_{\text {ave }}$ from experimental results $\left(\frac{\left|f_{\text {ave,num }}-f_{\text {ave, exp }}\right|}{f_{\text {ave, exp }}}\right)$ for the two microchannel heat sinks is less than $5 \%$. The maximum deviation of numerical $N u_{\text {ave }}$ from experimental results $\left(\frac{\left|N u_{\text {ave,enum }}-N u_{\text {ave,exp }}\right|}{N u_{\text {ave,exp }}}\right)$ for IMCHS-R with rib length $0.4 \mathrm{~mm}$ and MCHS is respectively less than $11.7 \%$ and $6 \%$.

\subsection{Velocity, pressure and temperature distributions}

Figures 4 and 5 respectively show velocity distributions and streamlines at the second microchamber in the plane of $z=0.25 \mathrm{~mm}$ as $R e=441$ where the flow direction is from left to right. As the flow passage becomes narrower or wider, the velocity increases or decreases 
due to the Bernoulli Effect. As the flow vertically impinges the rib and fin (between microchannels) tips, the stagnation zones to occur. As the flow passage becomes diverging, flow recirculation is formed at the lateral edge of rib. As water flows out from the narrow passage, flow recirculation is formed at the rib and fin wakes due to the sudden expansion of flow passage.

Figure 6 shows pressure fields at the second microchamber in the plane of $z=0.25 \mathrm{~mm}$ as $R e=441$. As pointed out by Chai et al. [3], there are three effects to determine the pressure distribution in microchamber with ribs, including Bernoulli effect, entrance and exit loss, and the stagnation or recirculation zone. The first two effects mainly caused by the changed flow passage, which has been studied by Xu et al. [5] and Hong and Cheng [12], and the last effect mainly results from the rib geometry. It can be seen that the recirculation at the fin wake makes the flow passage to diverge very slowly, which leads to the slow pressure drop. For the IMCHS-FT, as water flows in the space between the rib wake and microchannel inlet, the forward triangular rib causes the largest area of recirculation zone in the rib wake, resulting in the narrowest flow passage and the first rapid pressure drop. For the IMCHS-R and IMCHS-BT, the rectangular and backward triangular ribs not only result in the largest area of stagnation zone in the rib tip where the pressure is high and the velocity is very small, but also the sudden constriction flow passage in the inlet of rib gap which resulting in a rapid pressure drop as water enters. For the IMCHS-R and IMCHS-BT, there are two small recirculation zones to form at the lateral rib edges, especially for the backward triangular rib, which is wholly surrounded by very low velocity liquid. For the IMCHS-D and IMCHS-E, there is almost no stagnation or recirculation zone to develop in the microchamber, due to the 
gradually changed flow passage.

Figure 7 depicts the temperature distributions at the second microchamber in the plane of $z=0.25 \mathrm{~mm}$ as $R e=441$. It can be seen that there is much lower temperature gradient in the microchannel sidewall near the outlet of microchamber than that near the inlet. It indicates that the microchamber not only can interrupt the thermal boundary layer for the upstream microchannel, but also enhance mixing of cold and hot fluid there. For the IMCHS-R and IMCHS-BT, under the suppression due to the sudden constriction of flow passage, the cold water in the center and the hot water in the lateral sides can quickly mix with each other, leading to almost the same temperature in the rib gap. For the IMCHS-D, IMCHS-FT and IMCHS-E, the cold water in the center separates in the rib tip due to the acute angle and flows along the rib sidewalls. For the IMCHS-R and IMCHS-FT, the higher temperature in the large recirculation zone in the rib wake can be observed due to the very small velocity as shown in Figs. 4 and 5, which can deteriorate the mixing of water and the heat transfer performance.

\subsection{Local pressure drop and heat transfer characteristics}

Figure 8a shows the numerical results of local pressure $p_{\mathrm{x}}$ along $x$ direction as $R e=441$. It is noted that the interrupted microchannel heat sinks with ribs have higher pressure drop than the IMCHS and MCHS. Different rib geometry leads to different pressure drop. The IMCHS-R and IMCHS-BT show the largest pressure drop, while the other three present lower. For the MCHS, the pressure continuously drops along the flow direction, except a rapid decrease near the microchannel inlet due to the developing region. For the IMCHS, the pressure drop consists of not only the term along the microchannel, but also the term in the 
microchambers. As water flows into the microchamber from microchannel, the pressure drop becomes slow resulting from the expansion of flow passage, where the velocity slows down and the pressure builds up, which can withdraw part decreased pressure. As water flows into the microchannel from microchamber, the pressure drops more quickly, where water speeds up and consumes more pressure energy, resulting from the constriction of flow passage and redeveloping of laminar flow boundary layer. For the interrupted microchannels with ribs, the pressure drop still contains the term caused by the ribs. Different rib geometries lead to different diverging or converging flow passage between ribs, thus different pressure drop tendencies. The clearer display of pressure drop at the second microchamber is shown in Fig. $8 \mathrm{~b}$. The four vertical lines respectively represent the sides of microchamber beginning, rib tip, rib wake and microchamber end as shown in Fig. 4. As water flows into the rib region from the microchamber, the pressure drops quickly for the IMCHS-R and IMCHS-BT due to the sudden constriction of flow passage, while drops slowly for the IMCHS-D, IMCHS-FT and IMCHS-E due to the gradually changed flow passage.

Figure $9 \mathrm{a}$ shows the variation of local Poiseuille number $\left(f_{\mathrm{x}} R e\right)$ along the non-dimensionalized length $\left(x^{+}\right)$as $R e=441$. The $x^{+}$is defined as $x^{+}=x /\left(D_{\mathrm{h}} R e\right)$. For the interrupted microchannels, the $f_{\mathrm{x}} R e$ drops along the flow direction in the microchannel region and microchamber. As water flows into the next microchannel from microchamber, the $f_{\mathrm{x}} R e$ increases quickly and then drops down due to the redeveloping laminar boundary layer. Fig. $9 \mathrm{~b}$ shows the clearer variation of $f_{\mathrm{x}} R e$ at the second microchamber. As water flows into the microchamber from microchannel, the $f_{\mathrm{x}} R e$ drops without a sudden change, due to the gradual expansion of flow passage resulting from the recirculation zone developed at the fin wake. As 
water flows into the rib region from microchamber, there are rapid increases of $f_{\mathrm{x}} R e$ for IMCHS-R and IMCHS-BT due to the sudden constriction of flow passage, and continual drop of $f_{\mathrm{x}} R e$ for IMCHS-D, IMCHS-FT and IMCHS-E due to the gradually changed flow passage. As water flows out of the rib region, the $f_{\mathrm{x}} R e$ drops continually for all the interrupted microchannels. As water flows into the next microchannel, the $f_{\mathrm{x}} R e$ increases near the microchannels inlet and drops down along the microchannel.

Different microchannel heat sink geometry leads to different velocity distribution and pressure field, and thus different heat transfer performance. Fig. 10a shows the variation of temperature $T_{\mathrm{w}, \mathrm{x}}$ at the heat sink base $(z=0)$ along the flow direction as $R e=441$. It can be seen that for the interrupted microchannel heat sinks, the maximum temperature at the base is much smaller than MCHS, especially for the heat sinks with ribs, suggesting the better heat transfer performance. It is noted that for the IMCHS, the $T_{\mathrm{w}, \mathrm{x}}$ increases quickly as water flows into the microchamber from microchannel and drops rapidly as water flows into the next microchannel. The rapid increased temperature in microchamber region may burn the high heat flux equipment in actual applications. The deteriorated heat transfer performance in the microchamber region can solved by introducing the staggered ribs. The ribs in the microchamber can effectively suppresses the increase of $T_{\mathrm{w}, \mathrm{x}}$ in the microchamber region and even make the $T_{\mathrm{w}, \mathrm{x}}$ lower than the temperature in the upstream microchannel region as shown in Fig. 10a. The clearer local temperature variation of $T_{\mathrm{w}, \mathrm{x}}$ corresponding to the second microchamber region is shown in Fig. 10b. It can be seen that the temperature is much lower for the microchannel heat sinks with ribs than the MCHS and IMCHS. For the interrupted microchannel heat sinks with ribs, the $T_{\mathrm{w}, \mathrm{x}}$ just changes a little as water flows into the 
microchamber from microchannel, quickly decreases as water flows into the rib gap, maintains almost the same as water flows from the rib gap to the microchamber, and decreases a little as water flows into the next microchannel. The variation of $T_{\mathrm{w}, \mathrm{x}}$ in the rib gap depends on the geometry of flow passage. For the IMCHS-FT, the converging flow passage leads to the decrease of $T_{\mathrm{w}, \mathrm{x}}$. For the IMCHS-D and IMCHS-E, the first half converging and last half diverging flow passages lead to the first decrease and then increase of $T_{\mathrm{w}, \mathrm{x}}$. For the IMCHS-BT, the diverging flow passage leads to the increase of $T_{\mathrm{w}, \mathrm{x}}$.

Figure 11a shows the local Nusselt number $\left(N u_{\mathrm{x}}\right)$ along the non-dimensionalized length $\left(x^{*}\right)$ as $R e=441$. The $x^{*}$ is defined as $x^{*}=x /\left(D_{\mathrm{h}} \operatorname{RePr}\right)$. It can be noted that for the interrupted microchannels, the $N u_{\mathrm{x}}$ drops along the flow direction in the microchannel region and increases rapidly as water flows into the next microchannel due to the redeveloping of the thermal boundary layer. For MCHS, the $N u_{\mathrm{x}}$ continually drops along the flow direction; For IMCHS, the microchamber can prevent the drop of $N u_{\mathrm{x}}$ and make it to a new high level; For the interrupted microchannels with ribs, the rib not only prevents the deterioration of $N u_{\mathrm{x}}$ in the microchamber region in advance, but also improves the $N u_{\mathrm{x}}$ to a much higher level for the microchannel region. It can be seen that in the inlets of middle and rear microchannel regions, the $N u_{\mathrm{x}}$ for the interrupted microchannels with ribs are much higher than the IMCHS. The clearer $N u_{\mathrm{x}}$ variation corresponding to the second microchamber region is shown in Fig. 11b. It can be seen that for the IMCHS, the $N u_{\mathrm{x}}$ continually decreases as water flows into the microchamber from microchannel and starts to rise before water flows into the next microchannel. For the interrupted microchannel heat sinks with ribs, the $N u_{\mathrm{x}}$ starts to ascent as water flows into the microchamber. At the inlet of rib gap, the $N u_{\mathrm{x}}$ still increases due to the 
narrower flow passage. As water flows in the rib gap, the $N u_{\mathrm{x}}$ drops first for IMCHS-BT, second for IMCHS-R, and then IMCHS-D and IMCHS-E, but always rises for IMCHS-FT. These tendencies can be explained as follows: The continuous diverging flow passage and the recirculation formed at the lateral edge of rib make the $N u_{\mathrm{x}}$ drops first for IMCHS-BT; The continuous converging flow passage makes water continually impinge the sidewalls of rib to make the $N u_{\mathrm{x}}$ always increase for IMCHS-FT; The first half converging and last half diverging flow passages lead to the first increase and then decrease of $N u_{\mathrm{x}}$ for IMCHS-D and IMCHS-E. As water flows into microchamber from the rib gap, the $N u_{\mathrm{x}}$ decreases for the interrupted microchannel heat sinks with ribs, but the $N u_{\mathrm{x}}$ has begun to increase for IMCHS. Before water flows into the next microchannel, the $N u_{\mathrm{x}}$ starts to increase for the interrupted microchannel heat sinks with ribs. After a short distance away from the microchannel inlet, the $N u_{\mathrm{x}}$ reaches the maximum and then decreases along the flow direction.

\subsection{Overall pressure drop and heat transfer characteristics}

Figure 12 shows the relationship between average Poiseuille number $\left(f_{\text {ave }} R e\right)$ and Reynolds number $(R e)$ in the range of 187 to 715 . From Fig. 12a, it can be observed that with an increase of $R e$, the $f_{\text {app }} R e$ for the interrupted microchannels increase quickly and are obviously higher than those of MCHS. The $f_{\text {app }} R e$ of IMCHS shows lower values than that of MCHS at a very lower $R e$, but the increase velocity of $f_{\text {app }} R e$ with $R e$ for IMCHS is much higher than MCHS. For the interrupted microchannels with ribs, the increase velocity for $f_{\text {app }} R e$ is much higher than IMCHS, and IMCHS-R and IMCHS-BT show the two largest increase velocity and obviously higher than the other three. Among the interrupted 
microchannels with ribs, the IMCHS-D shows the lowest $f_{\text {app }} R e$. For $R e$ ranging from 187 to 715 , there is a $-6-23 \%$ increase in $f_{\text {app }} R e$ for IMCHS, there are $13-70 \%, 13-70 \%, 8-70 \%$, $3-40 \%, 5-44 \%$, and $6-44 \%$ increase in $f_{\text {app }} R e$ respectively for IMCHS-R, IMCHS-BT, IMCHS-D, IMCHS-FT and IMCHS-E, comparing with MCHS. Fig.12b shows the relationship between average Nusselt number $\left(N u_{\text {ave }}\right)$ and $R e$ ranging from 187 to 715 . It can be noted that with an increase of $R e$, the $N u_{\text {ave }}$ for the interrupted microchannel heat sinks increases rapidly and is obviously higher than those of MCHS. For the studied Re range, there is a $2-33 \%$ increase in $N u_{\text {ave }}$ for IMCHS, there are $30-57 \%, 25-55 \%, 24-54 \%, 24-53 \%$, and 28-57\% increase in $N u_{\text {ave }}$ for IMCHS-R, IMCHS-BT, IMCHS-D, IMCHS-FT and IMCHS-E, respectively, comparing with MCHS.

Figure 13 shows the performance evaluation criteria $(P E C)$ for such microchannel heat sinks in the Re range of 187 to 715 . It can be seen that, for the studied $R e$ range, the IMCHS-E shows the best heat transfer performance and IMCHS-BT shows the worst resulting from the rapid increase of $f_{\text {ave }}$ with $R e$. With an increase of $R e, P E C$ for IMCHS always increases, but $P E C$ for the interrupted microchannel heat sinks with ribs shows different tendencies. For IMCHS-R, PEC firstly increases and then reduces after about $R e=$ 450, indicating that it gradually lost its advantage used as an effective heat transfer enhancement method due to the higher $f_{\text {ave. }}$. For the other interrupted microchannels with ribs, PEC always increases with $R e$, but the increase velocity becomes slower than IMCHS-R. For the studied Re range, $P E C$ is $1.04-1.24$ for IMCHS, 1.25-1.33, 1.21-1.30, 1.22-1.38, 1.22-1.36, and 1.25-1.39 for IMCHS-R, IMCHS-BT, IMCHS-D, IMCHS-FT and IMCHS-E, respectively. 


\section{Conclusions}

Three-dimensional numerical simulations have been performed to study laminar flow and heat transfer in the interrupted microchannel heat sink with ribs in the transverse microchambers. Five different rib configurations are considered, including rectangular, backward triangular, diamond, forward triangular and ellipsoidal. With respect to the straight baseline microchannel, the new conclusions drawn in this paper are summarized as follows:

(1). The introduction of the staggered ribs into the transverse microchambers not only suppresses the heat transfer deterioration for IMCHS in the microchamber region, but also improves the heat transfer coefficient to a new higher level in the microchannel region. The heat transfer enhancement mainly attributes to the mixing of cold and hot water in microchamber and the much higher redeveloping level of thermal boundary layer in the microchannel regions.

(2). For the interrupted microchannel heat sinks with ribs, the velocity, pressure and temperature distributions in the microchamber are mainly under the influence of Bernoulli effect, entrance and exit geometry, and the developed stagnation or recirculation zone. The $f_{\mathrm{x}} R e$ drops but $N u_{\mathrm{x}}$ starts to ascent as water flows into the microchamber from microchannel. Both of them increase as water flows into the rib gap from microchamber and drop as water flows out. As water flows into the next microchannel, the $f_{\mathrm{x}} R e$ and $N u_{\mathrm{x}}$ increases near the microchannel's inlet and drops along the microchannel.

(3). For Reynolds number ranging from 187 to 715 , there are $30-57 \%, 25-55 \%, 24-54 \%$, $24-53 \%$, and $28-57 \%$ increase in the Nusselt number, while the friction factor increased by 
$13-70 \%, 13-70 \%, 8-70 \%, 3-40 \%, 5-44 \%$, and $6-44 \%$, respectively for IMCHS-R, IMCHS-BT, IMCHS-D, IMCHS-FT and IMCHS-E, comparing with MCHS. For the studied Reynolds number range, $P E C$ is $1.04-1.24$ for IMCHS, 1.25-1.33, 1.21-1.30, 1.22-1.38, 1.22-1.36, and 1.25-1.39 for IMCHS-R, IMCHS-BT, IMCHS-D, IMCHS-FT and IMCHS-E, respectively. 


\section{Acknowledgements}

The work was supported by the Engineering and Physical Sciences Research Council (EPSRC) of the UK through research grant (EP/L001233/1) and the National Natural Science Foundation of China (51576005). 


\section{References}

[1] Y.J. Lee, P.K. Singh, P.S. Lee, Fluid flow and heat transfer investigations on enhanced microchannel heat sink using oblique fins with parametric study, International Journal of Heat and Mass Transfer 81 (2015) 325-336.

[2] J. Zhang, Y. Zhao, Y. Diao, Y. Zhang, An experimental study on fluid flow and heat transfer in a multiport minichannel flat tube with micro-fin structures, International Journal of Heat and Mass Transfer 84 (2015) 511-520.

[3] L. Chai, G. Xia, M. Zhou, J. Li, J. Qi, Optimum thermal design of interrupted microchannel heat sink with rectangular ribs in the transverse microchambers, Applied Thermal Engineering 51(2013) 880-889.

[4] J.L. Xu, Y.H. Gan, D.C. Zhang, Microscale heat transfer enhancement using thermal boundary layer redeveloping concept, International Journal of Heat and Mass Transfer 48 (2005) 1662-1674.

[5] J.L. Xu, Y.X. Song, W. Zhang, Numerical simulations of interrupted and conventional microchannel heat sinks, International Journal of Heat and Mass Transfer 51 (2008) $5906-5917$.

[6] L. Chai, G.D. Xia, M.Z. Zhou, Numerical simulation of fluid flow and heat transfer in a microchannel heat sink with offset fan-shaped reentrant cavities in sidewall, International Communications in Heat and Mass Transfer 38 (2011) 577-584.

[7] L. Chai, G.D. Xia, L. Wang, Heat transfer enhancement in microchannel heat sinks 
with periodic expansion-constriction cross-sections, International Journal of Heat and Mass Transfer 62 (2013) 741-751.

[8] G.D. Xia, L. Chai, M.Z. Zhou, Effects of structural parameters on fluid flow and heat transfer in a microchannel with aligned fan-shaped reentrant cavities, International Journal of Thermal Sciences 50 (2011) 411-419.

[9] G.D. Xia, L. Chai, H.Y. Wang, Optimum thermal design of microchannel heat sink with triangular reentrant cavities, Applied Thermal Engineering 31 (2011) 1208-1219.

[10] Y.J. Cheng, Numerical simulation of stacked microchannel heat sink with mixing-enhanced passive structure, International Communications in Heat and Mass Transfer 34 (2007) 295-303.

[11] W.M. Abed, R.D. Whalley, D.J.C. Dennis, R.J. Poole, Numerical and experimental investigation of heat transfer and fluid flow characteristics in a micro-scale serpentine channel, International Journal of Heat and Mass Transfer 88 (2015) 790-802.

[12] F. Hong P. Cheng, Three dimensional numerical analyses and optimization of offset strip-fin microchannel heat sinks, International Communications in Heat and Mass Transfer 36 (2009) 651-656.

[13] A.J. Foong, N. Ramesh, Laminar convective heat transfer in a microchannel with internal longitudinal fins, International Journal of Thermal Sciences 48 (2009) 1908-1913.

[14] A. Ebrahimi, E. Roohi, S. Kheradmand, Numerical study of liquid flow and heat transfer in rectangular microchannel with longitudinal vortex generators, Applied Thermal Engineering 78 (2015) 576-583. 
[15] L. Chai, G.D. Xia, H.S. Wang, Numerical study of laminar flow and heat transfer in microchannel heat sink with offset ribs on sidewalls, Applied Thermal Engineering 92 (2016) 32-41.

[16] L. Chai, G.D. Xia, J. Qi, Experimental and numerical study of flow and heat transfer in trapezoidal microchannels, Heat Transfer Engineering 33 (2012) 972-981.

[17] A. Sakanova, C.C. Keian, J. Zhao, Performance improvements of microchannel heat sink using wavy channel and nanofluids, International Journal of Heat and Mass Transfer 89 (2015) 59-74.

[18] G. Xie, H. Shen, C.C. Wang, Parametric study on thermal performance of microchannel heat sinks with internal vertical Y-shaped bifurcations, International Journal of Heat and Mass Transfer 90 (2015) 948-958.

[19] F.P. Incropera, Liquid cooling of electronic devices by single-phase convection, Wiley, 1999.

[20] R.L. Webb, Performance evaluation criteria for use of enhanced heat transfer surfaces in heat exchanger design, International Journal of Heat and Mass Transfer 24 (1981) $715-726$.

[21] R. Karwa, C. Sharma, N. Karwa, Performance evaluation criterion at equal pumping power for enhanced performance heat transfer surfaces, Journal of Solar Energy 2013 (2013) 1-9. 


\section{Nomenclature}

$A$

contact surface area of water and silicon, $\mathrm{m}^{2}$

$c_{p}$

specific heat, $\mathrm{J} \cdot \mathrm{kg}^{-1} \mathrm{~K}^{-1}$

$D_{\mathrm{h}}$

hydraulic diameter, $\mathrm{m}$

$f$

friction factor

$h$

heat transfer coefficient, $\mathrm{W} \cdot \mathrm{m}^{-2} \cdot \mathrm{K}^{-1}$

$k$

thermal conductivity, $\mathrm{W} \cdot \mathrm{m}^{-1} \cdot \mathrm{K}^{-1}$

$L$

length of microchannel heat sink, $m$

$n$

local coordinate normal to the wall

$N u$

Nusselt number

$p$

static pressure, $\mathrm{Pa}$

PEC

Performance evaluation criteria

$q$

heat flux at the base of heat sink, $\mathrm{W} \cdot \mathrm{m}^{-2}$

Re

Reynolds number

$T$

temperature, $\mathrm{K}$

$T_{\mathrm{w}}$

temperature at the heat sink base, $\mathrm{K}$

$T_{\mathrm{f}}$

fluid temperature, $\mathrm{K}$

$u, v, w$

velocity, $\mathrm{m} \cdot \mathrm{s}^{-1}$

V

volume, $\mathrm{m}^{3}$

W

width of computational domain, $\mathrm{m}$

$x, y, z$

three coordinates shown in Fig. 1, m 


\section{Greek letters}

$\eta$

ratio

$\rho$

density, $\mathrm{kg} \cdot \mathrm{m}^{-3}$

$\mu$

dynamic viscosity, $\mathrm{Pa} \cdot \mathrm{s}$

Subscripts

0

based straight microchannel

ave

average

$\exp$

experimental

f

fluid, friction factor

h

heat transfer

in

inlet

$\mathrm{m}$

mean

num

numerical

out

outlet

S

silicon 


\section{Figure captions}

Fig.1 Microchannel heat sinks

Fig.2 Grid detail $(\mathrm{z}=0.25 \mathrm{~mm})$

Fig.3 Comparison of present numerical results with experimental data of Chai et al. $[3,7]$.

(a) $f_{\text {ave }}$ versus $R e$ and (b) $N u_{\text {ave }}$ versus $R e$

Fig.4 Velocity distribution $(R e=441, z=0.25 \mathrm{~mm})$

Fig.5 Streamline $(\operatorname{Re}=441, z=0.25 \mathrm{~mm})$

Fig.6 Pressure distribution $(R e=441, z=0.25 \mathrm{~mm})$

Fig.7 Temperature distribution $(R e=441, z=0.25 \mathrm{~mm})$

Fig.8 $\quad p_{\mathrm{x}}$ versus $x(R e=441)$

Fig.9 $f_{\mathrm{x}} R e$ versus $x^{+}(R e=441)$

Fig.10 $T_{\mathrm{w}, \mathrm{x}}$ versus $x(R e=441)$

Fig.11 $N u_{\mathrm{x}}$ versus $x^{*}(R e=441)$

Fig.12 Comparison of $f_{\text {ave }} R e$ and $N u_{\text {ave }}$ with $R e$. (a) $f_{\text {ave }} R e$ versus $R e$ and (b) $N u_{\text {ave }}$ versus $R e$

Fig.13 PEC versus $R e$ 
Fig. 1
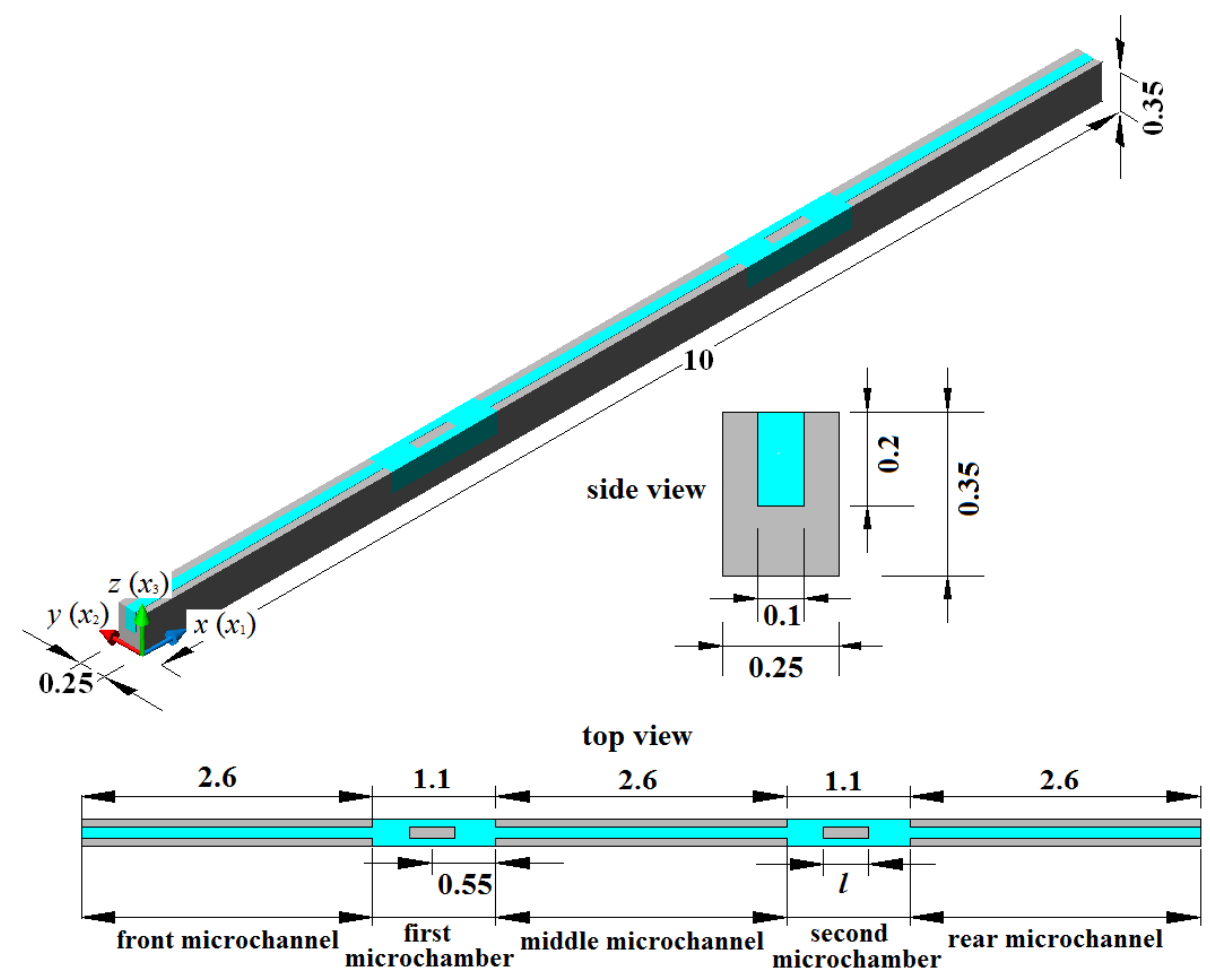

Unit: mm

(a)

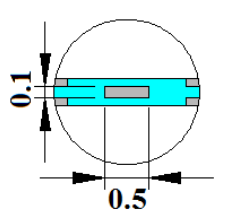

IMCHS-R

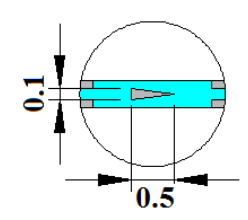

IMCHS-BT

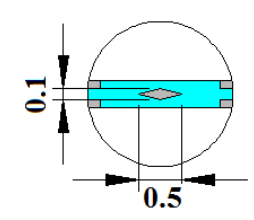

IMCHS-D

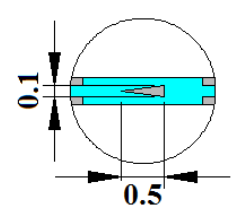

IMCHS-FT

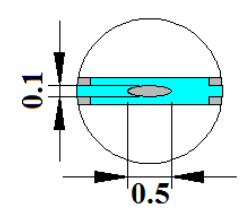

IMCHS-E

Unit: mm

(b) 
Fig. 2

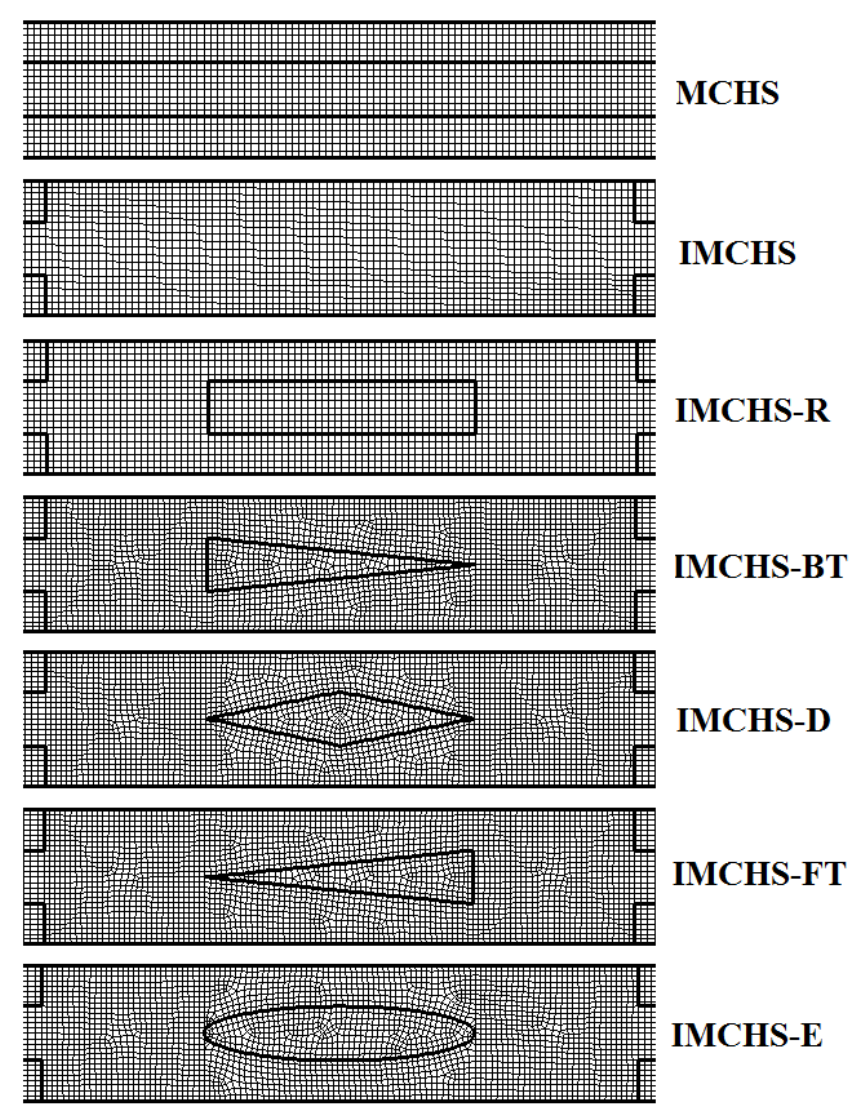


Fig. 3

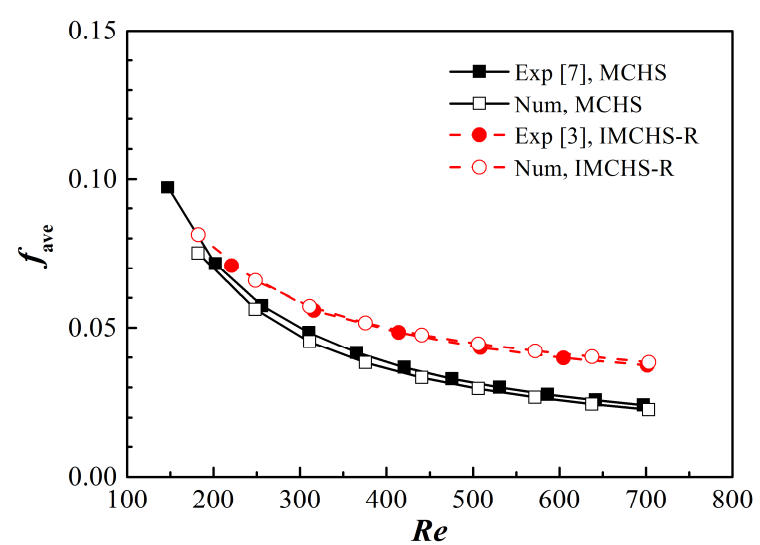

(a)

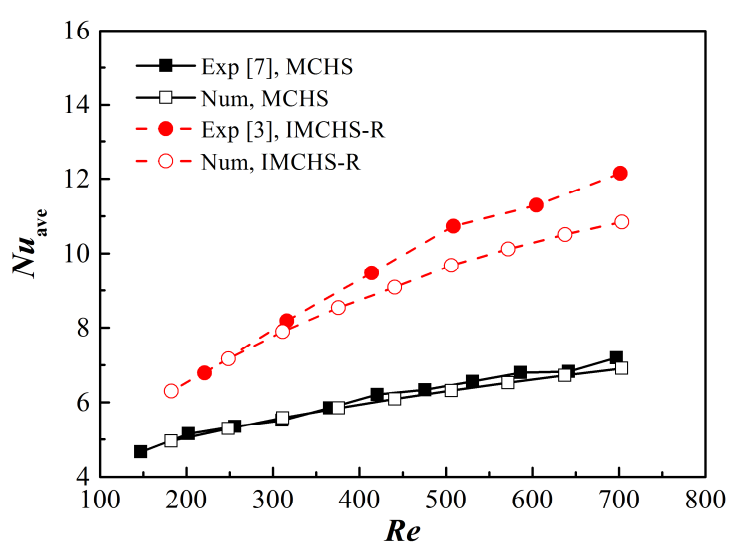

(b) 
Fig. 4

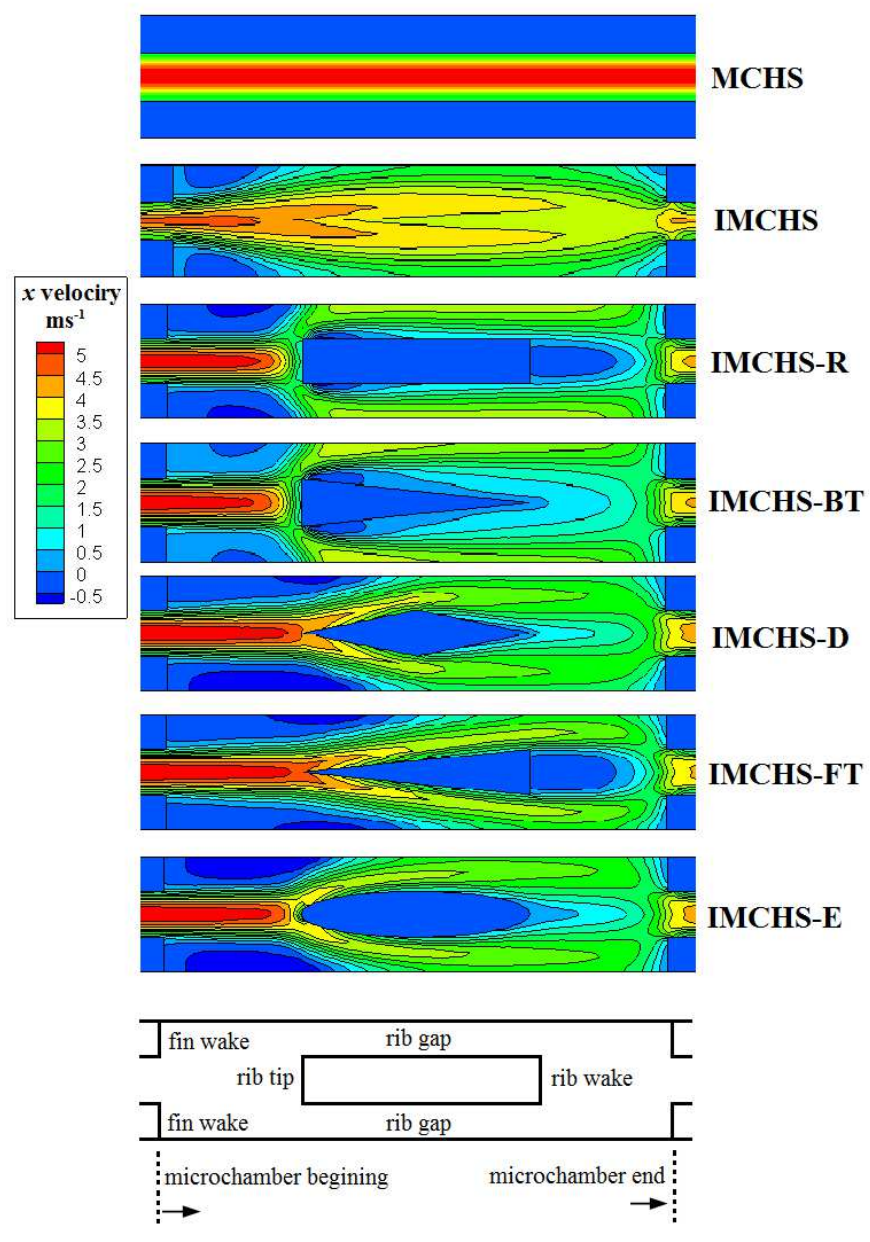


Fig. 5

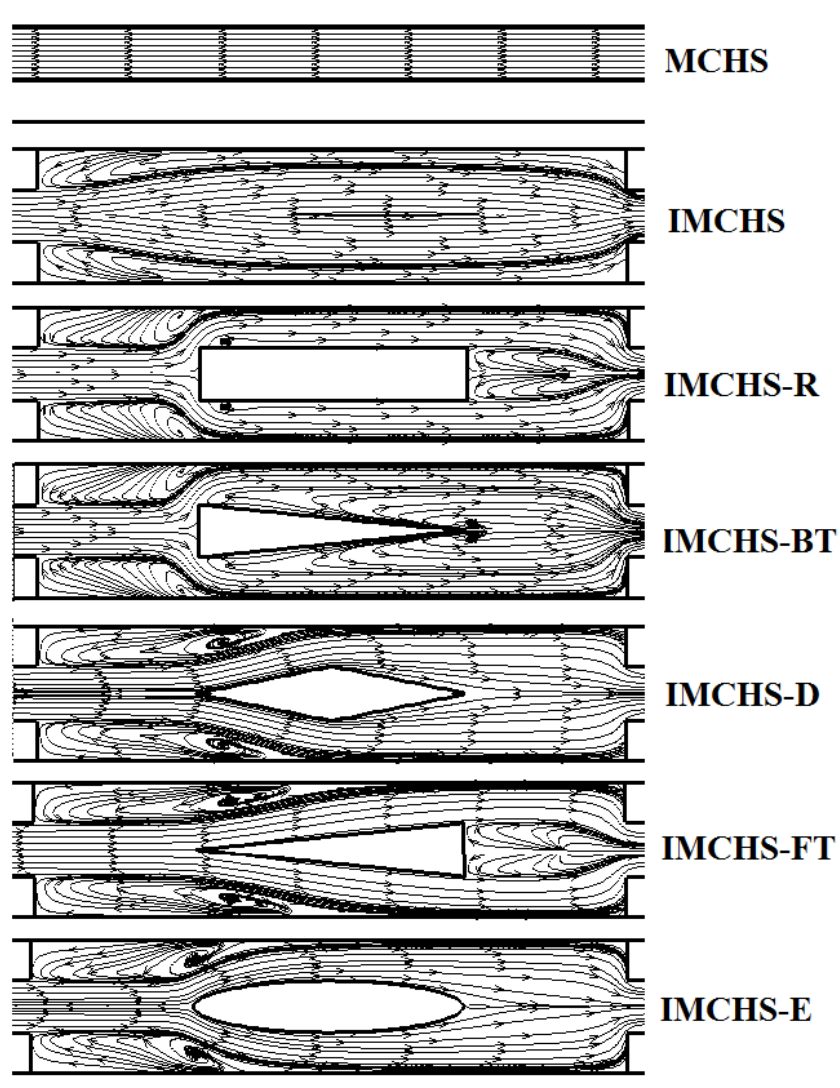


Fig. 6

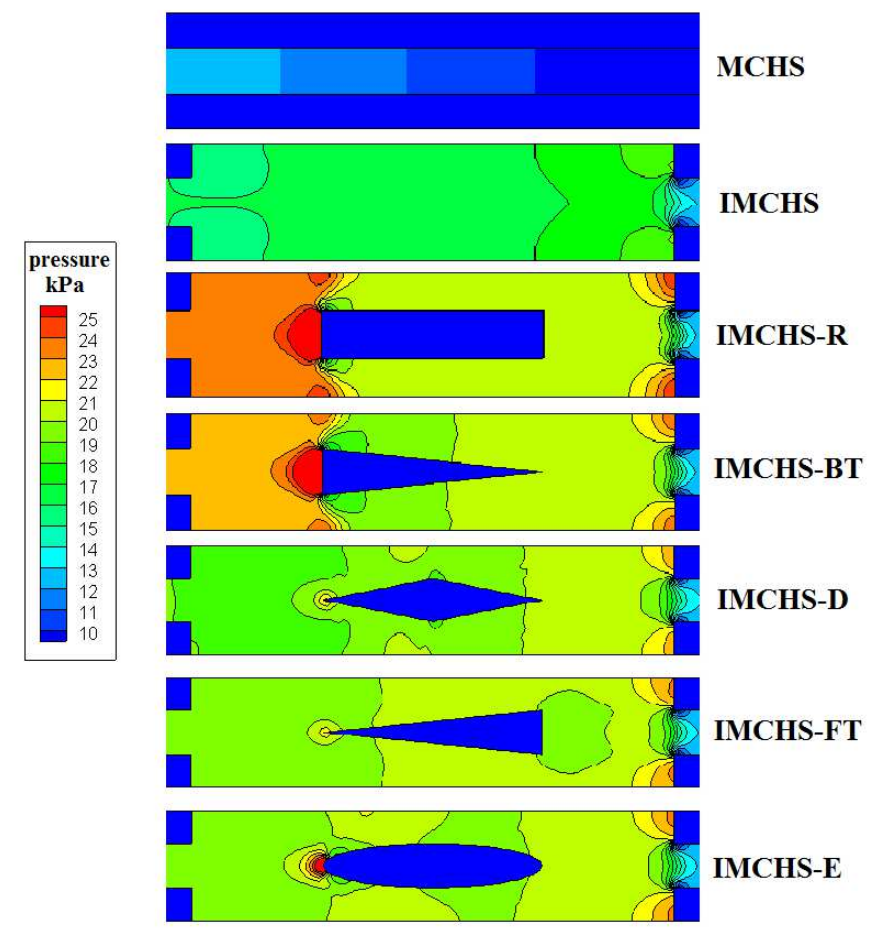


Fig. 7

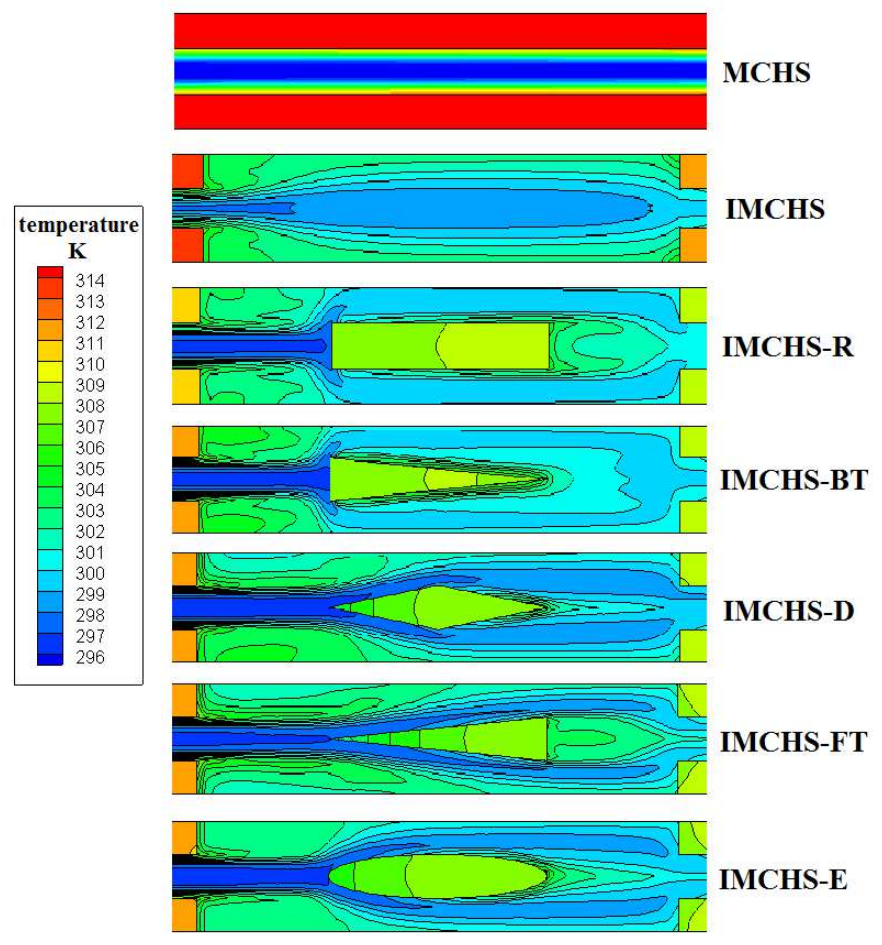


Fig. 8

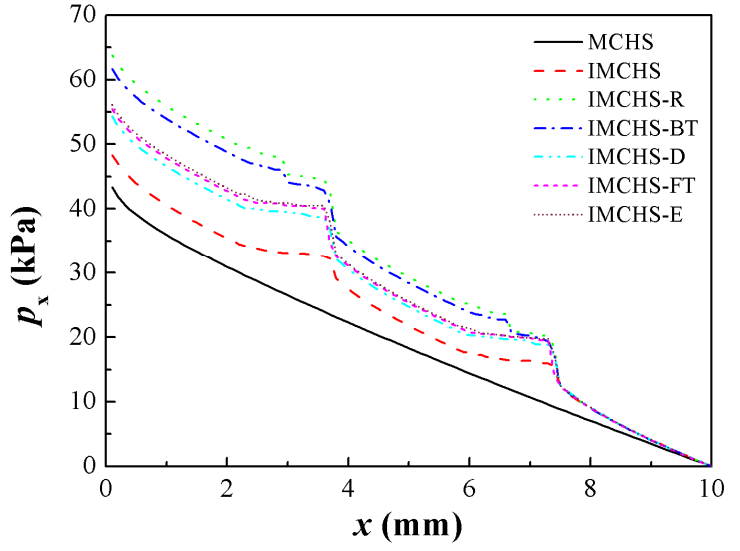

(a)

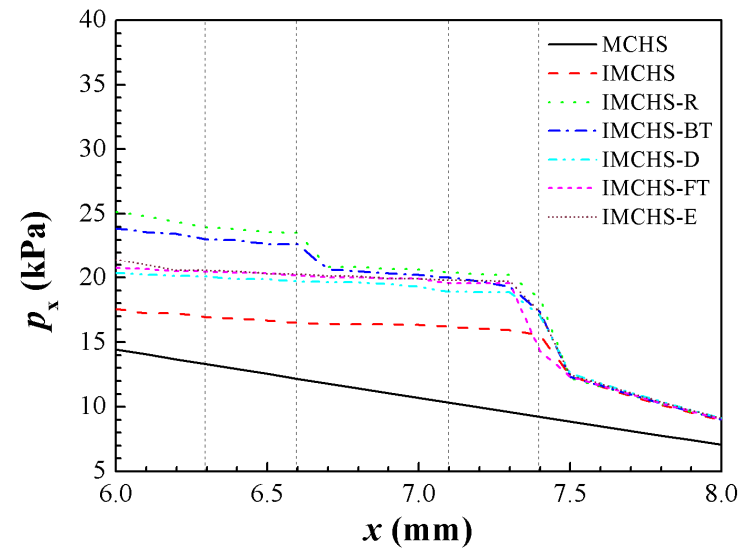

(b) 
Fig. 9

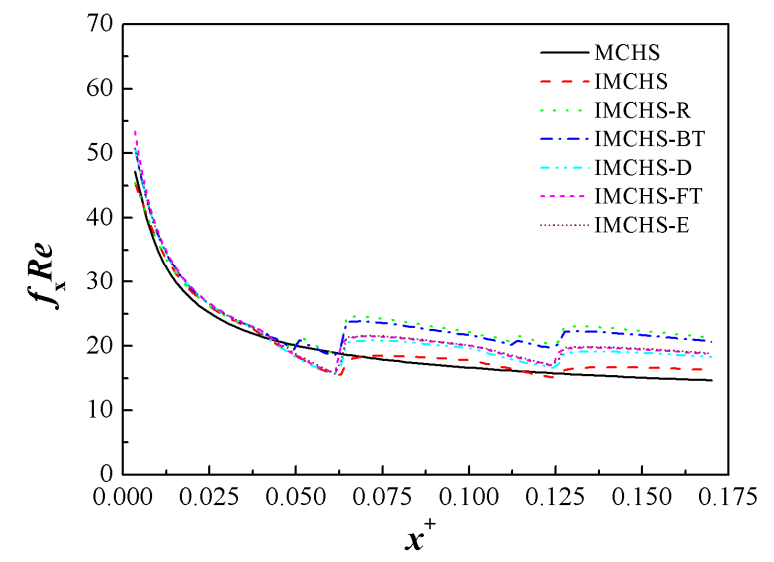

(a)

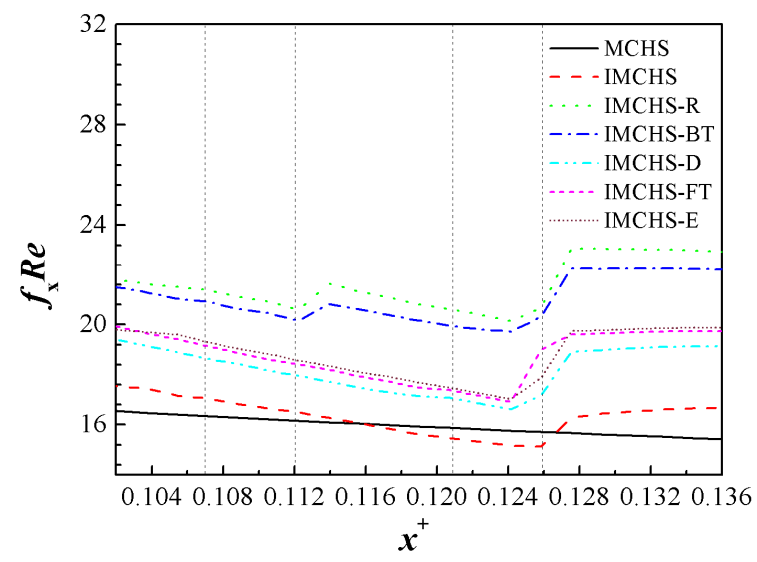

(b) 
Fig. 10

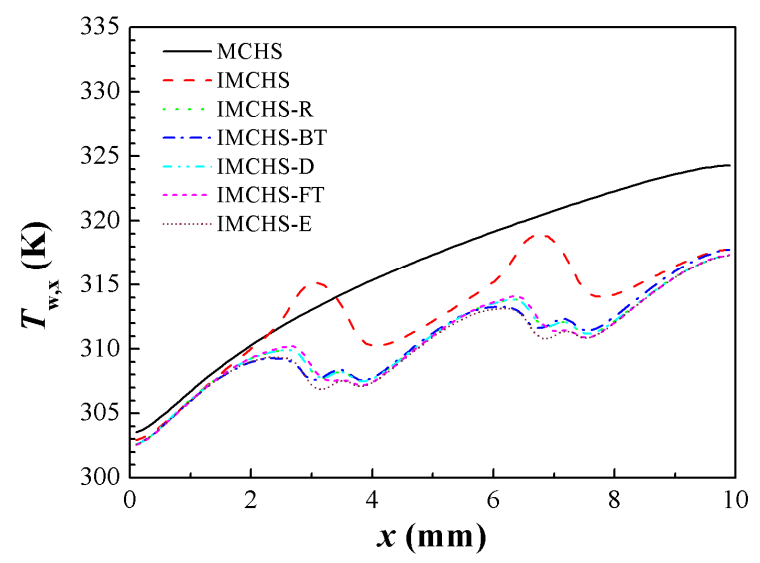

(a)

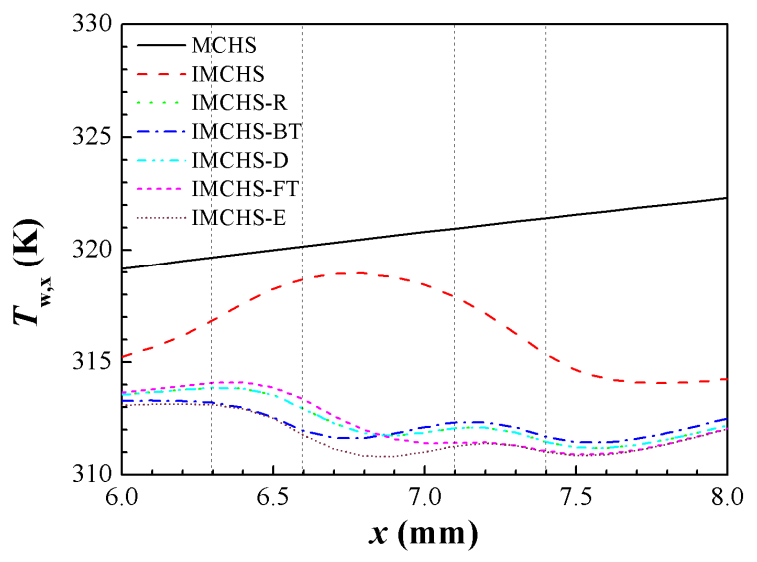

(b) 
Fig. 11

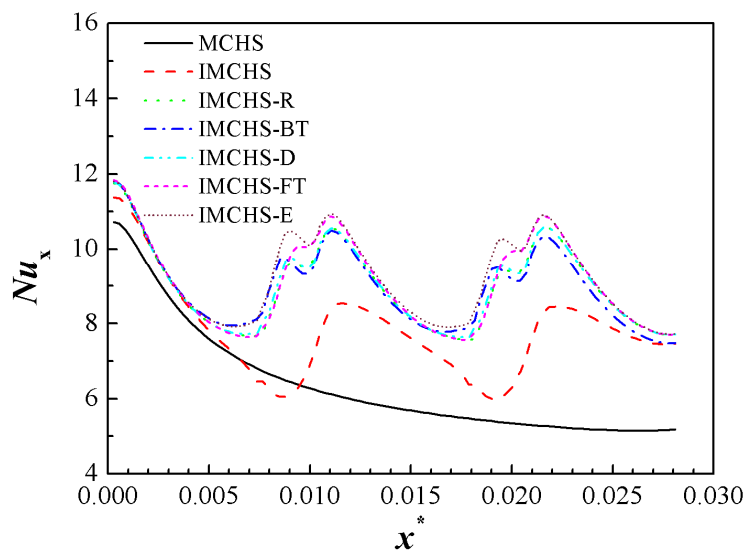

(a)

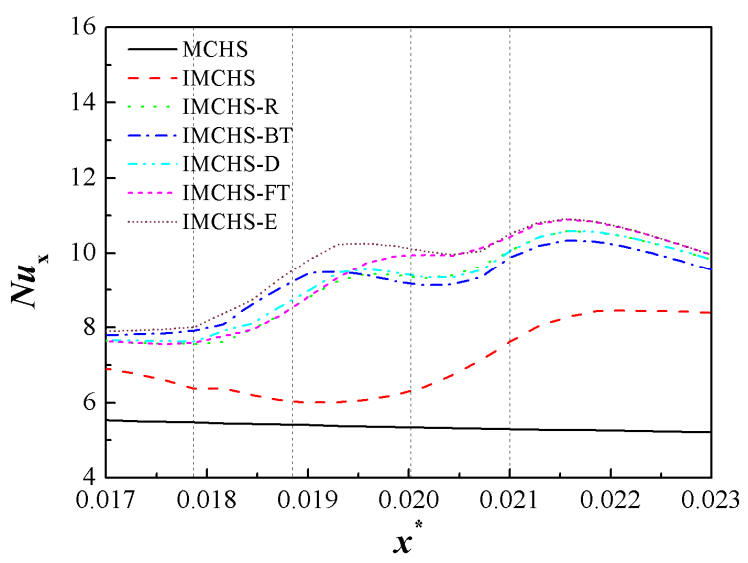

(b) 
Fig. 12

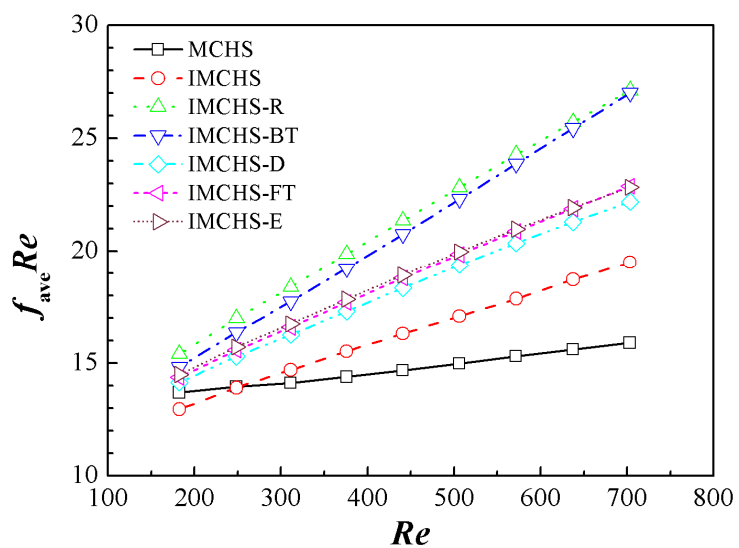

(a)

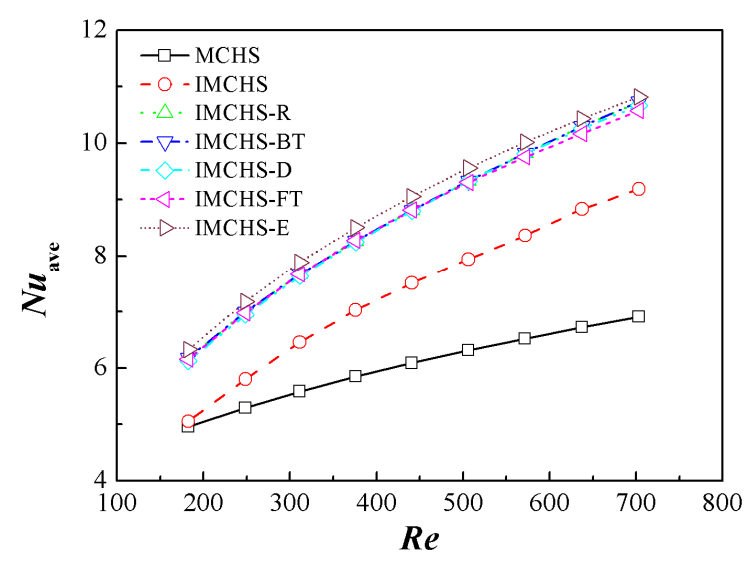

(b) 
Fig. 13

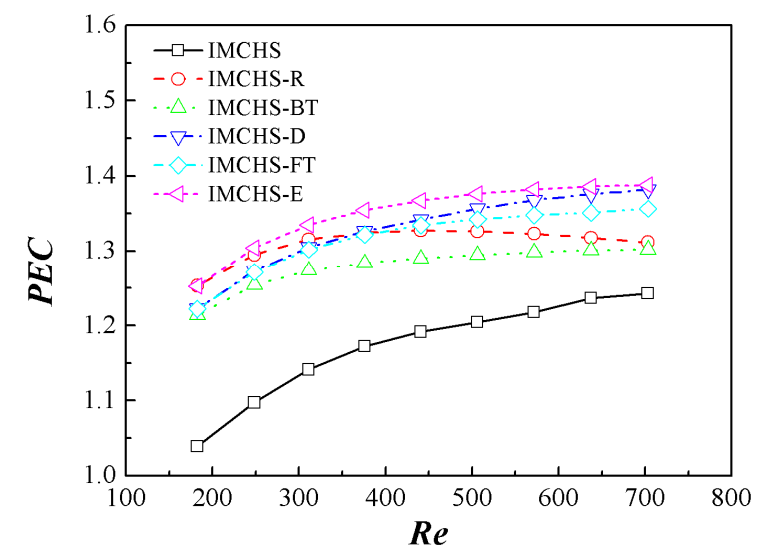

